\title{
Therapeutic Efficacy of Artemether-Lumefantrine for Treatment of Uncomplicated Plasmodium falciparum Malaria in Enugu, Nigeria
}

\author{
Ebere E Ayogu ${ }^{1,2 *}$, Chinwe V Ukwe ${ }^{1}$ and Emmanuel $\mathrm{O} \mathrm{Nna}^{3}$ \\ ${ }^{1}$ Department of Clinical Pharmacy and Pharmacy Management, Faculty of Pharmaceutical Sciences, University of Nigeria, \\ Nsukka, ${ }^{2}$ District Hospital, Nsukka, Ministry of Health, ${ }^{3}$ Safety Molecular Pathology Laboratory, Faculty of Health Sciences and \\ Technology, University of Nigeria, Enugu Campus, Enugu, Nigeria
}

*For correspondence: Email: ebereayogu@yahoo.com, ebereacademics@gmail.com; Tel: +234 8039307396

\begin{abstract}
Purpose: To review current therapeutic efficacy of artemether-lumefantrine (AL) for the treatment of uncomplicated Plasmodium falciparum malaria in patients in Enugu State, Nigeria.

Methods: One hundred and fifty four malaria patients from three different Local Government Areas (LGA) of Enugu State, southeastern Nigeria, that fulfilled the inclusion criteria were recruited through rapid diagnostic testing and blood film microscopy. The patients received a 3-day complete dose of $A L$ treatment; they were monitored and $3 \mathrm{~mL}$ of blood in EDTA bottle was sampled on days $0,3,7,14$ and 28. The samples were evaluated by real-time polymerase chain reaction (RT-PCR) for identification and quantification of Plasmodium falciparum. Clinical and parasitological responses were recorded and analyzed statistically.

Results: There was significant decrease $(p>0.002)$ in patient's temperature (from 40 to $37{ }^{\circ} \mathrm{C}$ ) from day 0 to day 28 . There was significant decrease $(p<0.001)$ between parasite mean density on day 0 and the values on days 3,7,14 and 28, with high prevalence of delayed parasite clearance. There was no early treatment failure, while 4 (3.4\%), 30 (25.4\%), and $84(75.1 \%)$ had late clinical failure, late parasitological response and adequate clinical and parasitological response, respectively.

Conclusion: Artemether - lumefantrine combination therapy reduced fever in malaria patients but failed to totally clear parasitemia density, indicating its reduced therapeutic efficacy in Enugu State, Nigeria.
\end{abstract}

Keywords: Plasmodium falciparum, Artemether-lumefantrine, Therapeutic efficacy, Malaria, Polymerase chain reaction

Tropical Journal of Pharmaceutical Research is indexed by Science Citation Index (SciSearch), Scopus, International Pharmaceutical Abstract, Chemical Abstracts, Embase, Index Copernicus, EBSCO, African Index Medicus, JournalSeek, Journal Citation Reports/Science Edition, Directory of Open Access Journals (DOAJ), African Journal Online, Bioline International, Open-J-Gate and Pharmacy Abstracts

\section{INTRODUCTION}

Widespread resistance of malaria parasites to commonly available anti-malarial drugs has necessitated countries to review and deploy new anti-malarial drug policies to ensure effective case management [1]. In 2005 Nigerian National Antimalarial Treatment Policy (NNATP) adopted artemether-lumefantrine (AL) as the first line drug for treatment of uncomplicated malaria in Nigeria. Although, several studies [2-4] done with children of 5 years and below have shown very good efficacy confirming the usefulness of $A L$, there is concern about the sustainability of $\mathrm{AL}$ against recrudescence and prevention against reinfection in older patients, and in areas such as Nigeria where prevalence of malaria transmission is very high with population at risk 
of malaria being $100 \%$ [5]. It is also not clear as to the duration of time for which $\mathrm{AL}$ will protect a patient from being re-infected by a new parasite bite.

The aim of the study was to review the current therapeutic efficacy of $\mathrm{AL}$ against uncomplicated $P$. falciparum malaria in older patients in Enugu, Nigeria.

\section{EXPERIMENTAL}

\section{Study design and population}

This prospective study was conducted in three hospitals from three different Local Government Areas in Enugu state namely; Nsukka (District Hospital Nsukka), Igbo-Eze North (Bishop Shanahan Hospital, Enugu-Ezike), and Nkanu East (Cottage Hospital Ugbuawka). Enugu state is located in South Eastern Nigeria. Enugu is in the malaria endemic zone and can be characterized by a stable perennial transmission of malarial infection throughout the year [6]. This study was conducted from January 2012 to March 2013 year.

One hundred and fifty four (154) patients who presented with symptoms compatible with acute uncomplicated malaria like headache and temperature of $37.5^{\circ} \mathrm{C}$ and above in the past two days, with positive malaria test and were above 6 years were enrolled. They were all out-patients who had not taken any antimalarial for the past 2 weeks. Patients who had history of serious side effect to the study drugs or were immunecompromised were not enrolled. A written informed consent was obtained from each eligible participant or parents/guidance in-case of children prior to enrollment. Following clinical diagnosis by a physician, patients were given tablets of $\mathrm{AL}$ according to Table 1 [7].

Each tablet of $\mathrm{AL}$ contains $20 \mathrm{mg}$ of artemether and $120 \mathrm{mg}$ of lumefantrine. Since participants were out-patients, a compliance form designed to help the patient take appropriate dose at the right time was given to each patient along with drug and was submitted on the next follow up visit. This was used to access patient compliance.

\section{Health research ethics approval}

The research methods of this study were screened and approved by the . University of Nigeria Teaching Hospital Health Research Ethical Committee (UNTH-HREC) Enugu with approval no. NHREC / 05 / 01 / / 2008B FWA00002458 - IRB00002323 and from the hospital administrations of each study Hospital. The study was carried out according to WHO 2002 guideline [8].

\section{Sample collection and laboratory procedure}

Screening was done by a Health Officer in an outpatient setting to identify patient's eligibility. Patient's demographic data and clinical findings during screening were retrieved. Finger prick blood was obtained for rapid malaria detection test whereby $3 \mathrm{ml}$ of venous blood samples were collected in EDTA bottles from patients that tested positive on day 0 before treatment with $A L$. The bottles were carefully labeled and identified. Blood samples were transported to Safety Molecular Pathology Laboratory within $5 \mathrm{~h}$ of collection. Patients were then monitored and followed up for 28 days. The follow up blood samples and temperature readings were obtained on days 3, 7, 14 and 28 in the hospital, after AL treatment for molecular analysis [9].

\section{Extraction of DNA}

$P$. falciparum genomic DNA was extracted from blood collected in EDTA bottles by Saponin heamolysis [10]. The principle is that saponin lyses red blood cell to release malaria parasite which is collected by centrifugation. Boiling and washing helped to yield more malaria rich genome for PCR amplification.

\section{Plasmodium falciparum amplification and quantification by nested PCR}

A set of HPLC purified primers, were used for nested PCR in Applied Biosystem 2720 thermal cyclers (Applied Biosystems, Singapore. Serial No. 272S1132046). A commercially optimized 2x PCR master mix (Promega UK) was used in setting up a $25 \mu$ reaction volume.

Table 1: Course of artemether-lumefantrine treatment

\begin{tabular}{|c|c|c|c|c|c|c|c|}
\hline \multirow{3}{*}{$\begin{array}{l}\text { Body } \\
\text { weight (kg) }\end{array}$} & \multirow[t]{3}{*}{ Age (years) } & \multicolumn{6}{|c|}{ Duration (days/time) } \\
\hline & & \multicolumn{2}{|c|}{1} & \multicolumn{2}{|c|}{2} & \multicolumn{2}{|c|}{3} \\
\hline & & $\mathrm{Oh}$ & $8 \mathrm{~h}$ post & am & $\mathrm{Pm}$ & $\mathrm{Am}$ & $\mathrm{pm}$ \\
\hline $5-14$ & $<3$ & $1 \mathrm{tab}$ & 1 tab & $1 \mathrm{tab}$ & $1 \mathrm{tab}$ & $1 \mathrm{tab}$ & $1 \mathrm{tab}$ \\
\hline $15-24$ & $>3-8$ & $2 \mathrm{tab}$ & $2 \mathrm{tab}$ & $2 \mathrm{tab}$ & $2 \mathrm{tab}$ & $2 \mathrm{tab}$ & $2 \mathrm{tab}$ \\
\hline $25-34$ & $>9-14$ & 3 tab & 3 tab & 3 tab & 3 tab & 3 tab & $3 \mathrm{tab}$ \\
\hline$>34$ & $>14$ & 4 tab & 4 tab & 4 tab & 4 tab & 4 tab & 4 tab \\
\hline
\end{tabular}


The first Round PCR used primers rPLU5 forward primer 5'-CCTGTTGTTGCCTTAAAC TTC and rPLU6 backward primer - 5'TTAAAATTGTTGCAGTTAAAAC at final concentrations of $300 \mathrm{nM}$ to amplify 1200 bp genus (Plasmodium) specific target of $18 \mathrm{~S}$ ribosomal RNA gene. The thermal profile for first Round PCR was initial denaturation of $95{ }^{\circ} \mathrm{C}$ for $10 \mathrm{~min}$, 35 cycles of $94{ }^{\circ} \mathrm{C}$ for $45 \mathrm{~s}, 58^{\circ} \mathrm{C}$ for $30 \mathrm{~s}, 72{ }^{\circ} \mathrm{C}$ for $30 \mathrm{~s}$ and final extension of $72{ }^{\circ} \mathrm{C}$ for $5 \mathrm{~min}$.

Second Round PCR reactions were set up using the first Round PCR product and primers - R2, rFAL 1 is forward primer 5' TTAAACTGGTTT GGGAAAACC and rFAL 2 is backward primer 5'ACACAATGAACTCAATCA TGA specific to $P$. falciparum for amplifying $205 \mathrm{bp}$ amplicon of the same gene at final concentration of $400 \mathrm{nM}$ in 25 $\mu \mathrm{l}$ reaction volumes [11]. The thermal profile for second round PCR was initial denaturation of 95 ${ }^{\circ} \mathrm{C}$ for $10 \mathrm{~min}, 35$ cycles of $94{ }^{\circ} \mathrm{C}$ for $45 \mathrm{~s}, 58{ }^{\circ} \mathrm{C}$ for $30 \mathrm{~s}, 72{ }^{\circ} \mathrm{C}$ for $30 \mathrm{~s}, 58{ }^{\circ} \mathrm{C}$ for $30 \mathrm{~s}$ and final extension of $72{ }^{\circ} \mathrm{C}$ for $5 \mathrm{~min}$. The second Round PCR products were visualized in $2 \%$ agarose gel using SYBER Safe or Web Green (Promega UK, WebScientific UK).

The study classified treatment responses based on Parasite clearance time (PCT) [12] according to World Health Organization (WHO) 1973 criteria, as; Sensitive (S), Mild resistance (RI), Moderate resistance (RII) and Severe resistance (RIII) [13].

Clinical responses on day 28 were classified based on the WHO 2002 criteria, as: Early Treatment Failure (ETF), Late Clinical Failure (LCF), Late Parasitological Failure (LPF) or Adequate Clinical and Parasitological Response (ACPR) [9]

\section{Statistical analysis}

Data generated were summarized in Excel and analyzed using both Graph Pad (Prism 5) and SPSS (version 16). Chi test was used to calculate the significant differences in temperature. Differences in parasitic densities of the various groups were analyzed using ANOVA and inter group comparison was done using Post Hoc test.

\section{RESULTS}

\section{Patient's demographic data}

Out of 160 patients enrolled, 6 were noncompliant with medication administration and were excluded. 101 were female while 53 were males. 31 (20.1\%), 45 (29.2 \%), 30 (19.4\%) and $48(31.1 \%)$ were within the ages of $6-12$ years, $13-25$ years, $26-40$ years and 41 years and above respectively.

\section{Clinical symptoms}

Clinical symptoms (headache and fever), reduced significantly with drug treatment. The highest body temperature was $40{ }^{\circ} \mathrm{C}$ while the least was $36.7^{\circ} \mathrm{C}$ on day 0 . The total number of patients with body temperature of between 37.1 and $40{ }^{\circ} \mathrm{C}$ were $110(71.4 \%)$ while $44(28.6 \%)$ had body temperature of $36.7-37{ }^{\circ} \mathrm{C}$ on day 0 . On day 3 , no patients had fever of $\geq 38{ }^{\circ} \mathrm{C}$ but 7 $(4.8 \%)$ had body temperature of $37.1-37.6{ }^{\circ} \mathrm{C}$. Four (3.7) patients on day 7, had body temperature of $38{ }^{\circ} \mathrm{C}$ with headache. There was significant decrease in patient's temperature from day 0 to day $28(p>0.002)$. The temperature readings on the follow up days are shown in Table 2.

\section{Parasiteamia density on follow-up days}

Out of the 154 patients sampled, 101 (78.6\%) were infected with Plasmodium falciparum, while $33(21.4 \%)$ were not. Mean parasite densities of $409 \pm 223,190 \pm 315,237 \pm 556,135 \pm 318$ and $193 \pm 371$ were noted at Days $0,3,7,14$ and 28 respectively.

Table 2: Temperature readings on follow-up days

\begin{tabular}{lccccc}
\hline Parameter & \multicolumn{5}{c}{$\mathbf{N ~ ( \% )}$} \\
\cline { 2 - 6 } & Day 0 & Day 3 & Day 7 & Day 14 & Day 28 \\
\hline$\leq 36{ }^{\circ} \mathrm{C}$ & 0 & $29(24.0)$ & $46(41.4)$ & $56(43.0)$ & $54(50.46)$ \\
$36.1-37^{\circ} \mathrm{C}$ & $44(28.6)$ & $88(73.0)$ & $63(56.7)$ & $58(54.2)$ & $52(48.5)$ \\
$37.1-38^{\circ} \mathrm{C}$ & $65(42.2)$ & $4(3.3)$ & $1(0.9)$ & $3(2.8)$ & $1(0.9)$ \\
$38.1-39^{\circ} \mathrm{C}$ & $36(23.4)$ & 0 & $4(3.6)$ & 0 & 0 \\
$39.1-40{ }^{\circ} \mathrm{C}$ & $9(5.8)$ & 0 & 0 & 0 & 0 \\
Total & 154 & 121 & 121 & 121 & 121 \\
Loss to follow-up & 0 & 7 & 10 & 14 & 14 \\
Eligibility & 154 & 114 & 111 & 107 & 107 \\
\hline
\end{tabular}


A comparison of the mean parasite densities on follow up days showed that there were significant decrease between day 0 and days $3,7,14$ and 28 $(p<0.001)$ while there were no significant reduction when day 3 was compared to day 7 and day 14 compared to day 28 ( $p>0.05)$. The results are shown in Table 3.

\section{Parasite clearance time}

The study showed that $65(58.0 \%)$ patients had delayed parasite clearance with PCT of more than 3 days on days 3 and 7, while 47 (42.0\%) had total parasite clearance on days 3 with PCT of $\leq 3$ days and were classified as sensitive (S) group. Out of the 65 patients 21 (18.2\%), 22 (19.6), and 22 (19.6\%) were classified as RI, RII and RIII respectively. Of the 22 patients in RIII, 4 $(6.06 \%)$ had no change in parasite density. The results are shown in Table 4.

\section{Clinical and parasitological PCR uncorrected responses on day 28 post-treatment}

For 28 days post treatment, 14 patients were lost to follow up resulting to $107(88.7 \%)$ eligible patient. There was no ETF, while 4 (3.4\%), 23 $(21.4 \%)$, and 80 (75 \%) had LCF, LPF and ACPR. Out of the $21.4 \%$ patients with LPF, 2.1 $\%$ (3) did not show any response, in terms of parasitaemia clearance/parasitological response as the parasite density remained the same after 3 days of treatment, while $16.5 \%$ (20) of the population had sub-optimal response with decreased parasitemia density on days 3 and 7 . Younger patients with age range between 6 and 15 had the highest percentage (87.8\%) contribution to ACPR. Other age ranges and their contribution to each treatment group with their area of habitation are shown in Table 5.

Table 3: Mean parasite density on follow-up days

\begin{tabular}{lcccc}
\hline $\begin{array}{l}\text { Follow-up } \\
\text { day }\end{array}$ & $\begin{array}{c}\text { Total parasite } \\
\text { density }\end{array}$ & $\begin{array}{c}\text { Mean density ( } \pm \\
\text { SD) }\end{array}$ & $\begin{array}{c}\text { Minimum } \\
\text { value }\end{array}$ & $\begin{array}{c}\text { Maximum } \\
\text { value }\end{array}$ \\
\hline Day 0 & 49225 & $406.82 \pm 223.69$ & 143 & 1475 \\
Day 3 & 23492 & $213.57 \pm 315.26^{*}$ & 0 & 600 \\
Day 7 & 22871 & $223.19 \pm 556.36^{*} \#$ & 0 & 1317 \\
Day 14 & 14038 & $133.70 \pm 318.39^{*}$ & 0 & 1839 \\
Day 28 & 19834 & $190.00 \pm 371.08^{*} \#$ & 0 & 1293 \\
\hline
\end{tabular}

*Mean difference is significance at $p<0.05$ when Day 0 was compared to Days 3,7,14 and 28; \#Mean difference is not significant at $p<0.05$ when Day 3 was compared to Day 7 , and Day 14 compared to Day 28

Table 4: Classification of treatment outcome based on parasite clearance time

\begin{tabular}{lcc}
\hline Variable & Frequency (\%) & $\begin{array}{c}\text { Classification of } \\
\text { patients }\end{array}$ \\
\hline $\begin{array}{l}\text { Delayed parasite clearance } \\
\text { Total parasite clearance on day 3 without }\end{array}$ & $65(58.0)$ & RI, RII, or RIII \\
recurrence & $47(42.0)$ & $\mathrm{S}$ \\
$\begin{array}{l}\text { Parasite clearance on day 3 with recurrence } \\
\text { on day 7 and 14 }\end{array}$ & $21(18.8)$ & $\mathrm{RI}$ \\
$\begin{array}{l}\text { Decrease in parasiteamia on day 3 } \\
\text { Increase in parasiteamia on day 3 }\end{array}$ & $22(19.6)$ & $\mathrm{RII}$ \\
\hline
\end{tabular}

Table 5: Clinical and parasitological PCR uncorrected responses on days 28 post treatment

\begin{tabular}{|c|c|c|c|c|c|c|}
\hline \multirow[t]{2}{*}{ Parameter } & \multirow[t]{2}{*}{ Total (\%) } & \multicolumn{3}{|c|}{ Age (years) } & \multicolumn{2}{|c|}{ Residence } \\
\hline & & $6-15$ & $16-30$ & $\geq 31$ & Urban & Rural \\
\hline No of patients & 121 & $38(31.4)$ & $53(43.8)$ & 30 (24.8) & $50(41.3)$ & $71(58.7)$ \\
\hline Lost to follow up & $14(11.5)$ & 5 (37.5) & $5(35.7)$ & $4(28.5)$ & $10(71.4)$ & $4(28.6)$ \\
\hline Eligibility & $107(88.4)$ & $33(30.8)$ & $48(44.8)$ & $26(24.3)$ & $40(37.4)$ & $67(62.6)$ \\
\hline ETF & 0 & 0 & 0 & 0 & 0 & 0 \\
\hline LCF & $4(3.4)$ & $1(3.0)$ & $3(6.2)$ & 0 & 0 & $4(6.0)$ \\
\hline LPF & $23(21.4)$ & $3(9.1)$ & 7 (14.6) & $13(50.0)$ & $13(32.5)$ & $10(15.0)$ \\
\hline Total failure & $27(25.5)$ & $4(12.1)$ & $10(20.8)$ & $13(50.0)$ & $13(32.5)$ & $14(20.9)$ \\
\hline ACPR & $80(75.0)$ & $29(87.9)$ & $38(79.2)$ & $13(50.0)$ & $27(67.5)$ & $53(79.1)$ \\
\hline
\end{tabular}

$A C P R$, adequate clinical and parasitological response; $L P F$, late parasitological failure; $L C F$, late clinical failure; $E T F$, early treatment failure 


\section{DISCUSSION}

In 2005, Nigeria adopted artemether lumefantrine as the new drug of choice for the treatment of uncomplicated malaria in all public health facilities and independent drug marketers (patent medicine vendors). The policy pronouncement was based on clinical trials carried out mainly with children in six Geopolitical zones in Nigerian in 2002 and 2004, and on the widely acclaimed efficacy of artemether lumefantrine globally [14]. It is imperative to note that the study used outpatients and did not administer drugs with any fatty food. This was done to create a scenario that depicted what is obtainable in real life, as almost all patients take their drug after normal meal without emphasis on the fatty aspect of the food. This would help to evaluate the efficacy of the drug without bias.

Patients treated in this study showed good responses clinically. $A L$ was able to clear fever totally in a higher percentage of the patients while very few had sub-optimal fever clearance at day 3 . On day 7 , a lesser percentage had body temperature of $>38^{\circ} \mathrm{C}$ with accompanying headache. Other follow up days maintained very high fever clearance. AL is known for high fever clearance rate and it is postulated that the rapid fever clearance is due to the activity of artemether which has a shorter half-life while the sustained action was by lumefantrine. The high fever clearance observed in this study supports the report of clinical trials done with children in Nigeria in 2002 and 2004 [14].

There is increasing evidence that parasite clearance time after ACT therapy is increasing in settings in Asia and Africa and clinical studies have identified delayed parasite clearance time as the most robust marker of artemisinin resistance [11, 15]. An accurate and reliable method for the early detection of artemisinin resistance has been the use of sampling multiple times a day at measured time points to estimate the rate of parasite clearance proposed [15]. However, this approach may be difficult to implement in settings of routine in vivo drug efficacy studies among outpatients. An alternative approach is to measure the detectable proportion of patients with parasitaemia after one, two or three days of the initiation of therapy $[16,17]$. Hence in this study, detectable proportion of patients with parasitaemia three days after the initiation of therapy was employed. Response to drug treatment based on parasite clearance was assessed using World Health Organization (WHO) 1973 criteria [12]. These criteria were used in this study because they best describe the nature of parasitaemia readings gotten from the quantitative $P C R$ results.

One third of the patient had total parasitaemia clearance on day 3 without recurrent parasitemia (sensitive). This group of patients was mainly younger patients indicating that $A L$ is more effective in younger patients than in older patients.

Our study showed high prevalence of patients with delayed parasite clearance after initiation of therapy suggesting emerging resistance to $A L$ in the study area. Among patients with delayed parasite clearance, some had decreased parasitaemia on days 3 and 7 showing slow parasitaemia clearance as against rapid parasitaemia clearance which could arise from novel parasite genotypes with reduced drug sensitivity. Such patients will experience delayed recovery from malaria attack following $A L$ treatment. This group of patients is not totally resistant to $A L$ therapy, but is an indicator that reveals dwindling efficacy of $A L$ in uncomplicated malaria treatment. Their pre-treatment parasite densities were observed to be higher than those obtained in the group that is sensitive to the drug. Our finding differs greatly from a study carried out with younger patients that revealed that out of 90 children treated with $\mathrm{AL}, 6(6.6 \%)$ had delayed PCT [18].

Another group of patients showed increased parasitaemia level on day 3 (severe resistance). This implies that parasite in this group of patients resisted treatment and is termed parasite resistance, which is ability of a parasite strain to survive and/or multiply despite the administration and absorption of drug in doses equal to or higher than those usually recommended but within the limits of tolerance of the subjects. The presence of parasitaemia on day 3 is most likely to be recrudescence than re-infection. However, it perhaps could be that patients with increased parasitemia level could have been exposed to new mosquito bites and had become re-infected. On the other hand a different school of thought may argue that the study drug should be able to protect the patient for at least 14 days post treatment against both re-infection and recrudescence. If this is true then, the presence of parasitaemia within this time range is considered as a discredit to $\mathrm{AL}$ therapy. With this observation, it has to be clearly stated as to what extent $A L$ drug protects a patient after initiation and completion of therapy. This treatment response was not associated to increased parasite density as almost more than half of the patients in this group had lower pre-treatment parasite density compared to other groups. Of 
great concern is the fact that all patient in this group except for one resulted in late parasitological failure.

It is of paramount importance to state that a small percentage of patients showed no response as their parasite densities remained the same at day 3 revealing another group that is resistant to $A L$ and had late parasitological failure even though there were no danger signs of severe malaria in them. A phenomenon that could lead to this observation suggesting artemisinin resistance is an increased propensity for these parasites to form "dormant" (or quiescent) rings under artemisinin exposure $[19,20]$. Parasites under this condition still avert the activities of $A L$ even when correct dosage is given. This could be the possible explanation for the above abnormality in parasitemia density post treatment with $A L$ in this study.

We observed that even though some of the patients showed sub optimal response and resistance to $A L$ treatment, we did not record any early treatment failure or late clinical failure as most patients with increased parasitemia level, post treatment, were asymptomatic. This could be explained by the fact that the study area Enugu South East Nigeria is situated in a malaria endemic area with high transmission rate thereby exposing individuals to high parasite load.

This study showed a low ACPR of $75 \%$, PCR uncorrected, at 28 days post treatment, $3.4 \%$ had LCF while $21.4 \%$ patients had LPF. One likely reason for this low ACPR could be not involving genotyping to differentiate between recrudescence and re-infection. It perhaps could mean that patients with increased parasitemia level could have been exposed to new mosquito bites and had become re-infected. Other factors that may be responsible for the low therapeutic efficacy observed in this study are; first, participants were $70 \%$ adult but children give better therapeutic response than adults. Secondly, it could arise from poor lumefantrine absorption as oral bioavailability of lumefantrine has been shown to be considerably reduced during the acute phase of malaria and also to vary from one individual to another [21]. Lastly, there is emerging resistance developing towards (AL) by Plasmodium falciparum, in Enugu State, South Eastern Nigeria. This agrees with a clinical report from Africa [22] and a study done in Enugu State, South Eastern Nigeria [23] showing reduced efficacy of $A L$ in parasitemia clearance. This observation could be attributed to some genetic variations like mutations, single nucleotide polymorphisms (SNPs) and copy number variation (CNV) in plasmodial genes leading to $A L$ resistance with significant decrease in plasmodial sensitivity.

\section{Limitations of the study}

This study has some limitations and the results were interpreted as such. The study did not differentiate recrudescence from re-infection, hence LCF, LPF and ACPR were uncorrected responses. The study used patients $>5$ years of age. The study also used outpatients who took their medications at home, and therefore, strict adherence to the recommended dosage regimen could not be ascertained.

\section{CONCLUSION}

Although it is encouraging to observe rapid fever elimination following the use of $A L$ therapy for patients with uncomplicated malaria in Enugu State, Nigeria, there was increase in parasite clearance time and high frequency of late parasitological failures among the patients, indicating reduction in the therapeutic efficacy of the combination therapy. Since $A L$ has been adopted as the first-line treatment for uncomplicated malaria in Nigeria's National Antimalarial Treatment Policy, government, policy-makers and researchers should focus on development and implementation of policies, strategies and interventions that will prevent the development of resistance to $\mathrm{AL}$ therapy in order to sustain its efficacy.

\section{ACKNOWLEDGEMENT}

The authors wish to thank the malaria patients who volunteered to participate in this study, as well as the hospital administration and staff of the three hospitals used for the study.

\section{REFERENCES}

1. Bloland PB, Ettling M, Meek S. Combination therapy for malaria in Africa: hype or hope? Bulletin of the WHO 2000; 78(12): 1378-1388.

2. Yeka A, Dorsey G, Kamya MR, Talisuna A, Lugemwa M, Rwakimari JB, Staedke SG. Artemether lumefanthrine vs dihydroartemisinin-piperaquine for treating uncomplicated malaria: a randomized trial to guide policy in Uganda. PLoS One 2008; 3(6): 23902396.

3. Falade $C$, Makanga $M$, Premji $Z$, Ortmann CE, Stockmeyer M, de Palacios PI. Efficacy and safety of artemether-lumefanthrine (coartem) tablets (six dose regimen) in African infants and children with acute uncomplicated malaria. Trans $R$ Soc Trop Med Hyg 2005; 99: 459-469 
4. Zurovac D, Tibenderana JK, Nankarbiwa J, SsekitoolekoJ, Njogu NJ, Rwakimari JB Meek S, Talisuna A, Snow RW. Malaria case management under artemether-lumefantrine treatment policy in Uganda. Malar J 2008; 7: 181-190

5. World Health Organization. World Malaria Report. 2012

6. Federal Ministry of Health, Nigeria. National Antimalarial Treatment Policy. May 2005

7. Dokomajilar C, Nsobya SL, Greenhouse B, Rosenthal PJ, Dorsey G. Selection of Plasmodium falciparum Pfmdr1 alleles following therapy with artemetherlumefantrine in an area of Uganda where malaria is highly endemic. Antimicrob. Agents Chemother 2006; 50: 1893-1895.

8. Sisowath C, Stromberg J, Martensson A, Msellem M, Obondo C, Bjorkman A, Gil JP: Invivo selection of Plasmodium falciparum Pfmdr1 $86 \mathrm{~N}$ coding alleles by artemether-lumefanthrine (coartem). J Infect Dis 2005; 191(6): 1014-1017

9. World Health Organization (2002). Monitoring antimalarial drug resistance. Report of a WHO consultation, Geneva Switzerland 3rd - 5th Dec. 2001.

10. Orjih A, Cherian P, AlFadhli S: Microscopic Detection of Mixed Malarial Infections: Improvement by Saponin Heamolysis. Med Princ Pract 2008; 17: 458-463

11. Price RN, Uhlemann AC, Brockman A, McGready R, Ashley E, Phaipun L, Patel R, Laing $K$, Looareesuwan S, White NJ. et al: Mefloquine resistance in Plasmodium falciparum and increased pfmdr1 gene copy number. Lancet 2004; 364: 438447.

12. Beshir KB, Sawa P, Drakeley CJ, Baidjoe AY, Mweresa $C K$, Yussuf RU, Omar SA, et al: Extended malaria parasite clearance time in African children following artemisinin-combination therapy enhances transmission to Anopheles mosquitoes. Malar J 2012; 11(Suppl 1): 020-021.

13. World Health Organization (1973). Chemotherapy of malaria and resistance to antimalarials. Report of a WHO Scientific Group Technical Report Series No 529.

14. Federal Republic of Nigeria. National Policy on Malaria Diagnosis and Treatment. March 2010
15. Flegg JA, Guerin PJ, White NJ, Stepniewska $K$. Standardizing the measurement of parasite clearance in falciparum malaria: the parasite clearance estimator. Malar J 2011; 10: 339-351

16. Stepniewska K, Ashley E, Lee SJ, Anstey N, Barnes KI, Binh TQ, D'Alessandro U, Day NP, de Vries PJ, Dorsey G. In vivo parasitological measures of artemisinin susceptibility. J Infect Dis 2010; 201: 570579

17. Das D, Price RN, Bethell D, Guerin PJ. Stepniewska K. Early parasitological response following artemisinincontaining regimens: a critical review of the literature. Malar J 2013; 12: 125-137

18. Sowunmi A, Adewoye E, Gbotsho G, Happi C, Sijuade A, Folarin A, Okuboyejo $T$, Michael $T$. Factors contributing to delay in parasite clearance in uncomplicated falciparum malaria in children. Malar $\mathrm{J}$ 2010; 9: 53-63

19. Teuscher F, Gatton ML, Chen P, Peters J, Kyle DE, Chenq Q. Artemisinin-induced dormancy in Plasmodium falciparum. Duration, recovery rates and implication in treatment failure. $J$ Infect Dis 2010; 202: 1362-1368.

20. Witkowski B, Lelievre J, Barragan MJ, Laurent V, Su Xz, Berry A, Benoit-Vical F. Increased tolerance to artemisininin Plasmoduim falciparum is mediated by a Quiscent mechanism. Antimicrob Agents Chemother 2010; 54: 1872-1877

21. Lim P, Alker A, Khim N, Shah NK, Icardona S, Doung S, Yi $P$, Bouth DM. Pfmdr 1 copy number and artemisinin derivatives combination therapy in falciparum malaria in Cambodia. Malar J 2009; 8: 1139.

22. Noedl $H$, Se $Y$, Schaecher $K$, Smith BL, Socheat $D$, Fukada MM: Evidence of artemisinin resistance in western Cambodia. N Engl J Med 2008; 359: 26192620

23. Ukwe CV, Ekwunife OI, Ayogu EE, Adibe MO: Cost Effectiveness of Artemether-lumefantrine, artesunateamodiaquine, and dihydroartemisinin-piperaquine in the treatment of uncomplicated malaria in Nigerian patients. Drug Invention Today 2010; 2(10): 429-432. 